\title{
Study on Vertical Dynamics Compensation for Wobbling Effect Mitiga- tion of Electrostatically Levitated Gyroscope
}

\author{
Jongmin Lee*, Hyungmin Song*, Sangkyung Sung**, Chang Joo Kim*** \\ Konkuk University, Seoul, Korea \\ Sangwoo Lee L*** $^{* *}$ \\ Agency for Defense Development, Daejon, Korea
}

\begin{abstract}
We present a study of vertical dynamics control of an electrostatically levitated gyro-accelerometer considering the wobbling effect and propose a tilt stabilization method with newly introduced control electrodes. Typically, a rotor in a vacuum rotates at high velocity, which may create a drift rate and lead to displacement instability due to the tilt angle of the rotor. To analyze this, first we set up a vertical dynamic equation and determined simulation results regarding displacement control. After deriving an equation for drift dynamics, we analyzed the drift rate of the rotor and the wobbling effect for displacement control quantitatively. Then, we designed new sub-electrodes for moment control that will decrease the drift amplitude of wobbling dynamics. Finally, a simulation study demonstrated that the vertical displacement control with the wobbling compensation electrodes mitigated the rotor's drift rate, showing the effectiveness of the newly proposed control electrodes.
\end{abstract}

Key words: gyroscope, levitated gyro-accelerometer, wobbling effect, drift rate, moment control

\section{Introduction}

Gyroscopes and accelerometers are used widely, depending on their performance, as inertial sensors. A conventional MEMS gyroscope is the most widely used inertial sensor because of its inexpensive price and reasonable performance. However, there are limitations to performance of conventional MEMS gyroscopes. To address this, many research studies have been conducted. Among these, the levitated multi-axis gyro-accelerometer ("levitated gyro") appears to go beyond the limitations of conventional MEMS gyroscopes, with inherent performance advantages [1-3].

The levitated gyro can measure three axis accelerations ( $\mathrm{x}$, $\mathrm{y}, \mathrm{z}$ ) and two angular rates ( $\mathrm{x}, \mathrm{y})$. By restoring displacement of the rotor generated by external acceleration, it is possible to measure the three axis accelerations. Also, using rebalancing of the torque of precession, two axis angular rates can be measured. For this purpose, the levitated gyro is usually operated in a vacuum. By increasing the rotating speed of a gyroscope, the precision and durability can also be much improved. For this reason, it is essential to control the displacement and high rotation velocity to operate a levitated gyro. However, the wobbling phenomenon occurs frequently when a body of revolution rotates at high angular velocity. This phenomenon is caused by the unbalanced mass distribution of the rotor. In case of the levitated gyro, it is an inevitable error factors during the manufacturing process. It is one of the risk factors threatening the stability and degrading sensor performance because a unit of the generated drift rate is equal to the output of angular velocity [3]. Thus, the wobbling effect needs to be further investigated to develop appropriate dynamic compensation.

Given this background, this paper presents the vertical dynamics control of an electrostatically levitated gyro
This is an Open Access article distributed under the terms of the Creative Commons Attribution Non-Commercial License (http://creativecommons.org/licenses/by$\mathrm{nc} / 3.0 /$ which permits unrestricted non-commercial use, distribution, and reproduction in any medium, provided the original work is properly cited.

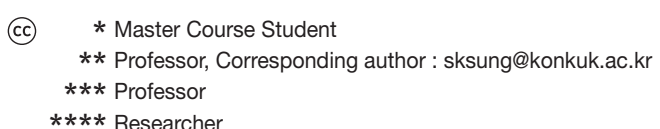


considering the wobbling effect and proposes an effective tilt stabilization method by newly introducing moment-control electrodes.

First, an angular drift rate model for the rotating rotor was developed with the assumption of point load initial mass unbalance. Then, for analyzing the wobbling effect of the rotor, parameters of mass-unbalance were assumed and an equation of drift rate was derived by developing the rotor's dynamic equation. In particular, a multi-variable capacitance model was developed to combine the derived drift angle with the previous vertical control model of a levitated gyroscope. The multi-variable capacitance model enabled formulating and designing a moment compensation algorithm in the tilt dynamics. Finally, a simulation study of the designed vertical dynamics control was done, considering the wobbling effect, which demonstrated that the proposed moment control strategy effectively mitigated the drift error of the tilt angle.

\section{Basic principle and modeling}

\subsection{Operational principle}

The basic principle was to detect the induced angle generated by the rotational moment of the electrically levitated rotor. An electrostatic force between the proof mass disk and electrodes generates vertical levitation and rotation of the rotor. While three axis accelerations are measured in controlling the displacement from the nominal point, two axis angular rates are measured with the precession angle, due to the rotor's induced torque.

Figure 1 shows the rotor and electrodes structure of the levitated gyro. The precession angle $\left(\theta_{x}\right)$ in sensing axis is caused by the induced torque as a vector product between the external angular rate $\left(\omega_{y}\right)$ and spinning rate $\left(\Omega_{z}\right)$. Typically, the precession is detected by the variation in capacitance generated by displacement of the rotor from sensing electrodes. Using restoring force as a control value, measurement and control are done at the same time. Equation (1) represents the moment equation to measure the external angular rate.

$$
M_{x}=I_{z} \cdot \omega_{y} \times \Omega_{z}
$$

where the moment $\left(M_{x}\right)$ generated by external angular rate $\left(\omega_{y}\right)$ is computed by the moment of inertia $\left(I_{z}\right)$ and the spinning rate $\left(\Omega_{z}\right)$ of the rotor. In (1), it is observed that the resolution of sensor is increased by spinning rate of the rotor. Usually, the spinning rate is expected to be about 10,000 rpm for a tactical-grade gyroscope in a MEMS device.

\subsection{Vertical dynamics modeling}

Fundamentally, a levitated gyro assumes a suspended rotating proof mass for its operation. For this, a dynamic equation is established about the vertical motion (usually denoted by the $\mathrm{z}$-axis) of the rotor[1].

$$
m \ddot{z}+b \dot{z}+k z=f_{z}+f_{d}
$$

where $m$ is the mass, $b$ the damping coefficient, $k$ the spring constant, $f_{z}$ the electrostatic force, $f_{d}$ the external force, and $z$ the displacement. Considering the electrostatic control voltage, the electrostatic force is calculated as

$$
f_{z}\left(z, U_{c}\right)=\frac{1}{2} \varepsilon A\left[\frac{\left(V_{B}+U_{c}\right)^{2}}{\left(d_{z}-z\right)^{2}}-\frac{\left(V_{B}-U_{c}\right)^{2}}{\left(d_{z}+z\right)^{2}}\right]
$$

where $V_{B}$ is the bias voltage, $U_{C}$ the control voltage, and $d_{z}$ the nominal point. Thus (2) can be depicted by the following equation, including the external acceleration of gravity.

$$
m \ddot{z}+b \dot{z}=\frac{1}{2} \varepsilon A\left[\frac{\left(V_{B}+U_{c}\right)^{2}}{\left(d_{z}-z\right)^{2}}-\frac{\left(V_{B}-U_{c}\right)^{2}}{\left(d_{z}+z\right)^{2}}\right]+m g
$$

In equation (4), the spring constant equals zero because the levitated gyro operates in a suspended state and the dynamic equation is established considering only gravity, with no additional external acceleration.
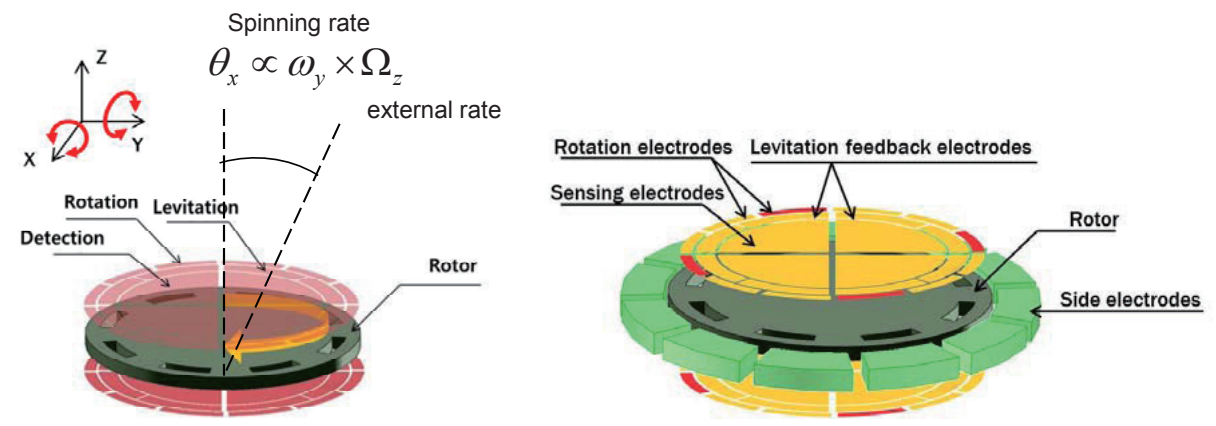

Fig. 1. Structure and working principle. 


\section{Analysis of the wobbling dynamics from mass unbalance}

\subsection{Drift rate characteristics}

The wobbling phenomenon in a levitation gyroscope is a periodic fluctuation of rotational proofmass from a nominal spinning plane. It is a characteristic motion when the spinning mass has a non-uniform mass distribution around the rotating disk or ring, which is amplified significantly at high spinning velocities. For analyzing the wobbling effect, an equation of the drift rate is developed using initial tilt angles and parameters that cause mass-unbalance [2].

Figure 2 shows the coordinate frames for equation development, where $\left(O-X_{o} Y_{o} Z_{o}\right)$ is the inertial reference frame, $(O-X Y Z)$ the angular momentum frame, and $\left(O-X_{e} Y_{e} Z_{e}\right)$ the body frame. The angular momentum frame is rotated with $\alpha$ about the $Z_{o}$-axis and with $\beta$ about the Xo-axis from the inertial reference frame. The body frame is rotated $\psi$ about the $Z$-axis and $\vartheta$ about the $X$-axis, and finally $\varphi$ from the angular momentum frame. Drift rate is derived by relating angular momentum and moment generated by the mass-unbalance through the frames. First, assume a massunbalance is placed on the $X_{e}$-axis of the body frame with a point load mass and distance from the center point. Then, comparing the angular momentum derivative transformed in the inertial reference frame with the moment from body to inertial frame provides the equivalent equation.

Let $\gamma_{1}, \gamma_{2}, \gamma_{3}$ be transforming components via the Euler angle from the inertial reference frame to the angular reference frame, as follows:

$$
\left\{\begin{array}{l}
\gamma_{1}=\cos \psi \cos \varphi-\cos \vartheta \sin \psi \sin \varphi \\
\gamma_{2}=\sin \psi \cos \varphi+\cos \vartheta \cos \psi \sin \varphi \\
\gamma_{3}=\sin \vartheta \sin \varphi
\end{array}\right.
$$

The initial angular momentum is converted into that in the inertial reference frame, which are represented by

$$
H=H_{0}\left(\sin \alpha \sin \beta \vec{X}_{0}-\cos \alpha \sin \beta \vec{Y}_{0}+\cos \beta \vec{Z}_{0}\right)
$$

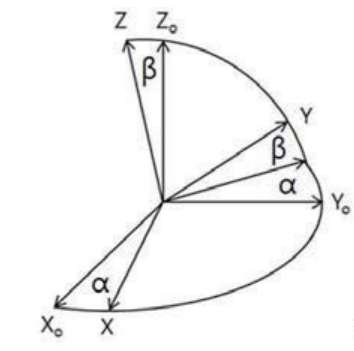

[Angular Reference Frame]

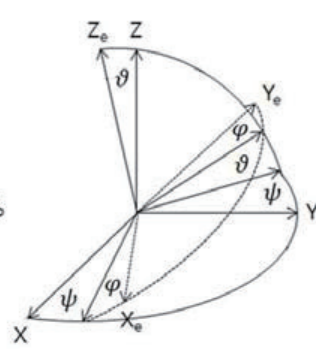

[Body Frame]
Fig. 2. Frames for inducing the drift rate. where $\vec{X}_{0}, \vec{Y}_{0}, \vec{Z}_{0}$ denote the unit vectors in the inertial frame. Then, equation (7) shows that moment converted from the body frame to the inertial reference frame.

$$
M=m_{e} g e_{e}\left[\begin{array}{l}
-\left(\gamma_{1} \sin \alpha+\gamma_{2} \cos \alpha \cos \beta-\gamma_{3} \cos \alpha \sin \beta\right) \vec{X}_{0} \\
+\left(\gamma_{1} \cos \alpha-\gamma_{2} \sin \alpha \cos \beta+\gamma_{3} \sin \alpha \sin \beta\right) \vec{Y}_{0}
\end{array}\right]
$$

where $m_{e}$ and $e_{e}$ represents mass and distance from the center point as parameters of mass-unbalance, respectively. Using rotational transformation in (5), it can be rearranged in the inertial reference frame. Through equations (5) (7), moment and angular momentum are expressed in the inertial reference frame. Thus, by comparing angular momentum derivative and moment from body frame, $\dot{\alpha}$ and $\dot{\beta}$ can be computed as follows.

$$
\dot{H}=M
$$

Using (6) and (7), equations for each unit vector $X_{o}, Y_{o}$ are arranged as

$$
\begin{aligned}
& -m_{e} g e_{e}\left(\gamma_{1} \sin \alpha+\gamma_{2} \cos \alpha \cos \beta-\gamma_{3} \cos \alpha \sin \beta\right)=\frac{d\left(H_{0} \sin \alpha \sin \beta\right)}{d t} \\
& m_{e} g e_{e}\left(\gamma_{1} \cos \alpha-\gamma_{2} \sin \alpha \cos \beta+\gamma_{3} \sin \alpha \sin \beta\right)=\frac{d\left(-H_{0} \cos \alpha \sin \beta\right)}{d t}
\end{aligned}
$$

Then, $\dot{\alpha}$ can be separated by multiplying by $\cos (\alpha)$ and $\sin (\alpha)$, then by eliminating $\dot{\beta}$. Equation (11) shows that $\dot{\beta}$ is eliminated and $\dot{\alpha}$ is derived as

$$
\dot{\alpha}=\varepsilon \frac{\cos \beta}{\cos \beta_{0}}[\sin \vartheta \sin \varphi-\cot \beta(\sin \psi \cos \varphi+\cos \vartheta \cos \psi \sin \varphi)]
$$

where a dimensionless eccentricity $\varepsilon\left(:=m_{e} g e_{e} / H_{0}\right)$ composed of parameters of mass-unbalance and initial angular momentum $\left(H_{0}\right)$ are newly introduced with the equality of

$$
H_{0} \cdot \dot{\alpha} \sin \beta=m_{e} g e_{e}\left(-\gamma_{2} \cos \beta+\gamma_{3} \sin \beta\right)
$$

Using the same method, $\dot{\beta}$ is induced as

$$
\dot{\beta}=\varepsilon_{0} \frac{\cos ^{2} \beta}{\cos \beta_{0}}(\cos \vartheta \sin \varphi \sin \psi-\cos \psi \cos \varphi)
$$

Consequently, through developing the equation, drift rates about $X_{o}, Y_{o}$ axis can be described as follows.

$$
\begin{aligned}
\omega_{X_{0}} & =\dot{\beta} \\
& =\varepsilon_{0} \cos \beta_{0}\left(\cos \vartheta_{0} \sin \mu t \sin v t-\cos v t \cos \mu t\right) \\
\omega_{Y_{0}}= & \dot{\alpha} \sin \beta \\
= & \varepsilon_{0} \sin \beta_{0}\left[\sin \vartheta_{0} \sin \mu t-\cot \beta_{0}\left(\sin v t \cos \mu t+\cos \vartheta_{0} \cos v t \sin \mu t\right)\right]
\end{aligned}
$$

In (14) and (15), $\beta_{0}$ and $\vartheta_{0}$ are initial tilt angles caused by the rotational components about the $X$-axis. $\psi$ and $\varphi$ 
are the angular rate before and after horizontal rotation is applied, where for simplicity a constant rotational velocity $v t$ and $\mu t$ can be assumed during short intervals, respectively.

\subsection{Wobbling effect analysis using system param- eters}

To analyze the drift rate, motion generated by massunbalance is considered with real physical parameters. Point load mass unbalance and distance from center point are set up and drift rate is calculated through the moment generated by mass-unbalance.

In Table 1, essential parameters are listed. Rotational velocity, mass of mass-unbalance, and distance of massunbalance are presented numerically. Rotational velocity is set up considering current technical levels in developing a levitated gyro structure and parameters about massunbalance are set up considering the errors generated through manufacturing processes.

Rotational velocity of the rotor is $\omega_{z}=\dot{\psi} \cos \vartheta+\dot{\varphi}$, expressed in summation of each rotational velocity term and a simulation was performed using 10,000 rpm. Depending on the distribution rate of $\dot{\psi}$ and $\dot{\varphi}$, a low-frequency component can be generated. From (15), the second term on the righthand side can be simplified to

Table 1. Parameters for inducing the drift rate.

\begin{tabular}{|l|l|}
\hline Rotational velocity $(\mu)$ & $10,000 \mathrm{rpm}$ \\
\hline Mass of mass-unbalance $\left(m_{e}\right)$ & $5.830 \times 10^{-7} \mathrm{~kg}(0.8 \%)$ \\
\hline Distance of mass-unbalance $\left(e_{e}\right)$ & $562.5 \mu \mathrm{m}(37.5 \%)$ \\
\hline Initial tilt angle & $0.03415^{\circ}$ \\
\hline
\end{tabular}

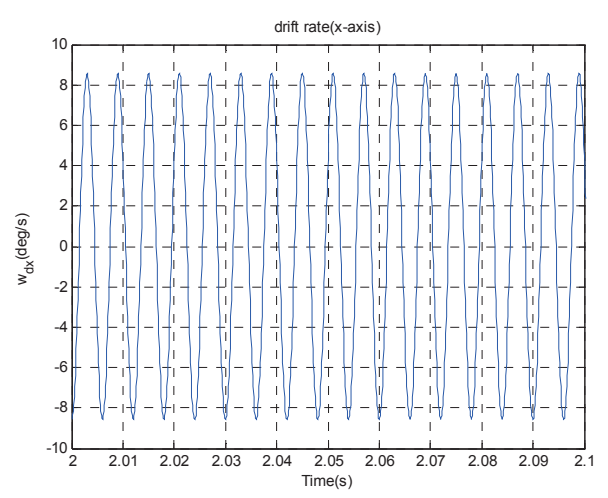

Fig. 4. Simulation results of drift rate about $\mathrm{X}$-axis.

$$
\frac{1-\cos \vartheta_{0}}{2} \sin (v-\mu) t
$$

where, considering $\vartheta_{0}$ is a very small deviation, the amplitude of the low-frequency component is as small as $10^{-10}$, and thus can be ignored.

Regarding the tilt angle about the $X$-axis, it is analyzed theoretically because the real configuration of the rotor and parameters should be considered. Thus, in this paper, the initial value is set up considering a maximum tilt angle of the rotor and suitable assumption about the rotational component. Note that the tilt angle is generated by the moment from mass-unbalance during the time that rotor moves from the stopper to a nominal point before operating the controller using the driving electrodes. Because displacement due to the tilt angle is calculated using the angular acceleration about the $X$-axis and the time that the rotor moves from the stopper to the nominal point, angular acceleration $\alpha_{x}$ is computed using

$$
M_{x}=I_{x} \alpha_{x}=m_{e} g r_{e}
$$

The displacement of the tilt angle can be computed numerically via simulation results using COMSOL Multiphysics, where a multi physics dynamic analysis for the

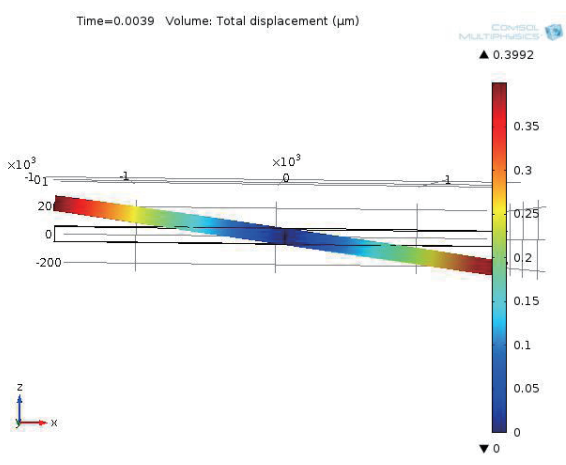

Fig. 3. Simulation (COMSOL) result of generated tilt angle.

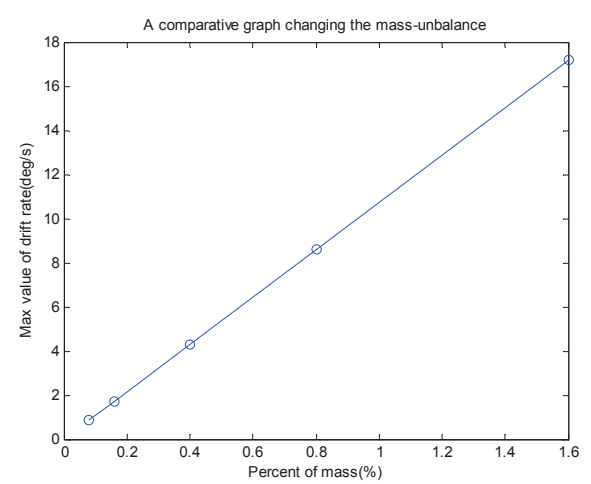


rotor dynamics with mass unbalance is simulated accurately. Fig. 3 shows the tilt angle where the initial unbalance is applied using the parameters in Table 1.

In Fig. 3, the tilt angle is computed by point load during the free falling time, $0.0039 \mathrm{~s}$ practically. As a result, the tilt angle is about $0.0161^{\circ}$, considering vertical displacement at the end of the rotor $(0.4216 \mu \mathrm{m})$ and the radius of the rotor, $1500 \mu \mathrm{m}$. It is appropriate that result from COMSOL is slightly smaller than the analytical calculation because friction and model uncertainty are neglected in the analytical computation. Finally, the drift rate in the time domain is calculated by applying the parameters of the real rotor and unbalance effects.

The drift rate in the time domain is provided in Fig. 4, where a sine wave with a $0.006 \mathrm{~s}$ period, that amounts to $10,000 \mathrm{rpm}$, is observed. The drift rate is proportional to the mass unbalance ratio, as observed in the right panel in Fig. 4 , which shows non-negligible magnitude of $\%$ for $0.1 \%$ mass irregularity.

Next, the initial tilt angle affecting drift rate is analyzed. With the small angle conditions on tilt angle, it is described as

$$
\left\{\begin{array}{l}
\sin \vartheta_{0}=\vartheta_{0}, \cos \vartheta_{0}=1 \\
\sin \beta_{0}=\beta_{0}, \cos \beta_{0}=1
\end{array}\right.
$$

Thus, (14) and (15) are further approximated into

$$
\begin{aligned}
& \omega_{X_{0}}=\varepsilon_{0}(\sin \mu t \sin v t-\cos v t \cos \mu t) \\
& \omega_{Y_{0}}=\varepsilon_{0} \beta_{0}\left[\vartheta_{0} \sin \mu t-\frac{1}{\beta_{0}}(\sin v t \cos \mu t+\cos v t \sin \mu t)\right]
\end{aligned}
$$

where the second term in the right hand side is more dominant that the first term in (20).

\section{Wobbling and multi-variable capacitance model}

\subsection{Multi variable capacitance model}

In this section, we describe the detailed detection of the rotor's motion, as affected by the drift rate with a wobbling effect. The periodic drift rate of the rotor inevitably generates a tilt angle. This effect on the capacitance model changes the electrostatic force. In equation (21), the basic equation for electrostatic force through a capacitor is shown:

$$
F=\frac{1}{2} \frac{\partial C}{\partial z} V^{2}
$$

In an ideal vertical levitation model, modeling is established using only the displacement as a variable and the other factors as constants. However, considering tilt angle, area is determined by tilt angles, so expression of area should be changed to include $\phi, \theta$. Thus, the single variable capacitance model needs to be converted into a multivariable capacitance model.

Figure 5 shows the inner and outer radii of the rotor and component of angle for calculating the overlap area [3]. Then, the capacitance of the rotor is determined by

$$
C_{1 t}(z, \phi, \theta)=\varepsilon_{0} \int_{0}^{\frac{\pi}{2}} \int_{R_{i}}^{R_{o}} \frac{r}{z+r k(\alpha)} d r d \alpha
$$

where $\mathrm{C}_{1 \mathrm{t}}$ represents capacitance in the first quarter electrode. A local displacement generated by tilt angle of the rotor is obtained as

$$
k(\alpha)=-\phi \cos \alpha+\theta \sin \alpha
$$

Through these equations, a multi-variable capacitance model is established, considering tilt angles.

$$
C_{1 t}(z, \phi, \theta)=\varepsilon_{0} \int_{0}^{\frac{\pi}{2}}\left\{\frac{R_{o}-R_{i}}{k(\alpha)}-\frac{z \ln \left(1+\frac{R_{o}}{z} k(\alpha)\right)}{k(\alpha)^{2}}+\frac{z \ln \left(1+\frac{R_{i}}{z} k(\alpha)\right)}{k(\alpha)^{2}}\right\} d \alpha
$$

Equation (24) can be further represented as a multi variable capacitance model as shown:

$$
C_{1 t}=\varepsilon_{0} \frac{\pi\left(R_{o}^{2}-R_{i}^{2}\right)}{4 z}+\varepsilon_{0} \frac{\left(R_{o}^{3}-R_{i}^{3}\right)(\phi-\theta)}{3 z^{2}}
$$

The expression is developed by using a Taylor series and an exponential function to obtained a final expression including only a first derivative term of $\phi, \theta$. Tilt angles are too small, so higher derivative terms of $\phi, \theta$ are effectively ignored. Using the above result, equation (26) represents the overall vertical dynamics control model.

$$
m \ddot{z}+b \dot{z}=\frac{1}{2} \varepsilon\left[\begin{array}{l}
\left(V_{B}+U_{C}\right)^{2}\left[\frac{\pi}{4} \cdot \frac{\left(R_{f o b}^{2}-R_{f b i}^{2}\right)}{\left(z_{0}-z\right)^{2}}+\frac{2}{3} \cdot \frac{\left(R_{f b o}^{3}-R_{f b i}^{3}\right) \cdot(\phi-\theta)}{\left(z_{0}-z\right)^{3}}\right] \\
-\left(V_{B}-U_{C}\right)^{2}\left[\frac{\pi}{4} \cdot \frac{\left(R_{f o b}^{2}-R_{f b i}^{2}\right)}{\left(z_{0}+z\right)^{2}}+\frac{2}{3} \cdot \frac{\left(R_{f b o}^{3}-R_{f b i}^{3}\right) \cdot(\phi-\theta)}{\left(z_{0}+z\right)^{3}}\right]
\end{array}\right]+m g
$$

where $R_{f b o}, R_{f b i}$ are the outer radius and inner radius of the driving electrode, respectively. Equation (26) shows the force
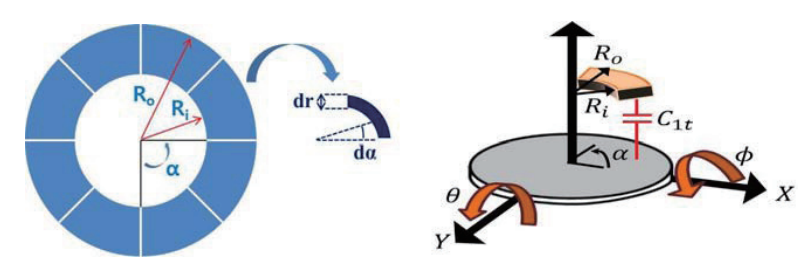

Fig. 5. Inner and outer radii of rotor and angular component. 
equation with a 'plus' sign for $\phi, \theta$ in the first quadrant. Equally, expression in the other quadrants can be determined by alternately changing the sign of the tilt angles. The established multi-variable capacitance model is used for the analysis of nominal vertical rebalance control and additional moment compensation for performance enhancement.

\subsection{Wobbling effects on vertical rebalance control}

This section discusses the wobbling effect due to mass unbalance in the vertical rebalancing control of the rotor. For this, nominal displacement control is designed and feedback control simulation is applied to the vertical dynamic model, considering both single and multi-variable capacitance cases. First, for designing a nominal controller, the nonlinear equation is linearized as

$$
m \ddot{z}+b \dot{z}=k_{z} z+k_{v} U_{c}
$$

where coefficients are obtained by partial differential terms of variable $z$ and $U_{C}$.

$$
k_{z}=\frac{2 \varepsilon A V_{B}^{2}}{z_{0}^{3}}, k_{v}=\frac{2 \varepsilon A V_{B}}{z_{0}^{2}}
$$

Applying the physical parameters, it can be determined that $k_{z}=4.7667$ and $k_{v}=1.1859 \times 10^{-5}$ because $V_{B}=1.2058 \mathrm{~V}$, $A=624784.2 \times 10^{-12} \mathrm{~m}^{2}$, and the initial gap $z_{0}=3 \mu \mathrm{m}$. With the linearized dynamic model, a simple PID controller is designed with control gain $K_{p}, K_{i}$, and $K_{d}$ of $0.6 \times 10^{6}, 1.0 \times 10^{8}$, and $1.0 \times 10^{3}$, respectively, providing a sufficient stability margin. Additionally, for practical application, the nominal rebalancing control needs to be combined with feed-forward control for initial excitation that drives the proof mass into the nominal dynamic equilibrium point. Then, a combined simulation is performed to verify the transient response for both linear and non-linear dynamics models. Fig. 6 demonstrates the sequential control strategy and transient response of the PID controller, based on the linearized model, successfully stabilizes the nonlinear dynamics model.

In Fig. 6, the right panel proposes the sequential control for initial excitation to an equivalent position, then application of the designed rebalance control for a full non linear dynamic model. For the initial levitation, it is designed such that only the upper driving electrodes are used to induce upward forces to suspend the rotor and then the rebalance controller is switched to operate around the nominal equivalent point. The right panel in Fig. 6 demonstrates initial excitation at $\mathrm{t}=$ $1 \mathrm{~s}$ from $\mathrm{z}=-2 \mu \mathrm{m}$ and immediately applies rebalance control at around $t=1.008 \mathrm{~s}$ with a successful convergence to the nominal position (i.e., $\mathrm{z}=0 \mu \mathrm{m}$ ). The integration time in the numerical simulation is $10^{-5} \mathrm{~s}$.

Under the same feedback control scenario, wobbling dynamics is considered to estimate the drift rate of the spinning rotor. AS in the simulation, initial mass unbalance, and tilt angle are applied in the disturbance form at $\mathrm{t}=1.1$ s (Fig. 7). Simultaneously, during rebalance control, the electrostatic force in each quadrant electrode is perturbed to disturb the spinning rotor's vertical displacement. It can be observed that amplitude of drift is $\sim 2.65 \times 10^{-2} \mu \mathrm{m}$.

\section{Wobbling mitigation design with a mo- ment compensator}

In the previous section, it was demonstrated that even though the average dynamics converges to the nominal position of levitation, a larger mass unbalance will cause unstable drift motion during gyroscope operation.

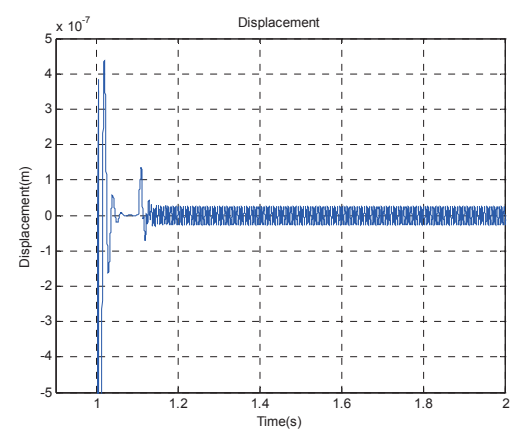

Fig. 7. Displacement of mass-unbalance rotor.
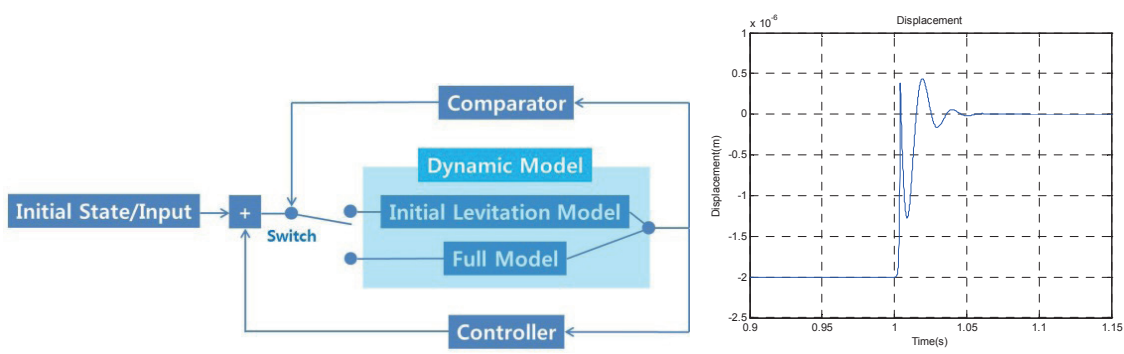

Fig. 6. Simulation result to assess the performance of the controller. 
Furthermore, the inherent angular drift rate can introduce characteristic sensor performance degradation, such as bias error. Simple vertical dynamics control cannot control the induced drift rate. To resolve the angular drift rate, novel electrodes for vertically asymmetric and unidirectional moment control using electrostatic forces are newly suggested in this section.

The main factor causing drift rate is the non-negligible third-order force term in the vertical dynamic equation in (26). In the case of the model considering tilt angles, the signs of $\phi, \theta$ are commutatively applied with (first quadrant $(+\phi,+\theta)$, third quadrant $(-\phi,-\theta))$ to the electrode placed diagonally. Using this characteristic, a complementary moment can be calculated. Thus, the correction electrode is designed to reduce the moment, considering the size and applied voltages of the sub-electrodes.

In Fig. 8, the left figure shows a layout of sub-electrodes with existing driving and detection electrodes. The right panel in Fig. 8 shows moments generated by driving and the newly designed sub-electrodes. As is known from the figure, subelectrodes are located on the bottom side only and generates moment to reduce the error factor with $U_{C} . \Delta M_{1}$ and $\Delta M_{1}^{\prime}$ denote the moment generated by the driving electrodes and the moment generated by the sub-electrodes, respectively. $N U_{c}$ denotes the newly proposed compensation electrodes.

Next, the principle to correct the error factor will be introduced by developing the equation. Moment generated by upper driving electrodes at the (I III) quadrant pair is calculated with equation (29).

$$
\begin{aligned}
& \frac{1}{2} \varepsilon R_{c 1}\left[\begin{array}{l}
-\left(V_{B}+U_{C}\right)^{2}\left[\frac{\pi}{4} \cdot \frac{\left(R_{f b o}^{2}-R_{f b i}^{2}\right)}{\left(z_{0}-z\right)^{2}}+\frac{2}{3} \cdot \frac{\left(R_{f b o}^{3}-R_{f b i}^{3}\right) \cdot(\phi-\theta)}{\left(z_{0}-z\right)^{3}}\right] \\
+\left(V_{B}+U_{C}\right)^{2}\left[\frac{\pi}{4} \cdot \frac{\left(R_{f b o}^{2}-R_{f b i}^{2}\right)}{\left(z_{0}-z\right)^{2}}-\frac{2}{3} \cdot \frac{\left(R_{f b o}^{3}-R_{f b i}^{3}\right) \cdot(\phi-\theta)}{\left(z_{0}-z\right)^{3}}\right]
\end{array}\right] \\
& =\varepsilon R_{c 1}\left[-\left(V_{B}+U_{C}\right)^{2}\left[\frac{2}{3} \cdot \frac{\left(R_{f b o}^{3}-R_{f b i}^{3}\right) \cdot(\phi-\theta)}{\left(z_{0}-z\right)^{3}}\right]\right]
\end{aligned}
$$

The first term, which is not affected by tilt angles, is cancelled and only the second term remains. Thus, the moment generated by all driving electrodes in the (I III) quadrant is calculated as follows.

$$
\begin{aligned}
\Delta M_{1}= & R_{c 1} \varepsilon\left(V_{B}-U_{c}\right)^{2}\left[\frac{2}{3} \cdot \frac{\left(R_{f b o}^{3}-R_{f b i}^{3}\right) \cdot(\phi-\theta)}{\left(z_{0}+z\right)^{3}}\right] \\
& -R_{c 1} \varepsilon\left(V_{B}+U_{c}\right)^{2}\left[\frac{2}{3} \cdot \frac{\left(R_{f b o}^{3}-R_{f b i}^{3}\right) \cdot(\phi-\theta)}{\left(z_{0}-z\right)^{3}}\right]
\end{aligned}
$$

In (30), all the moment generated by upper and lower driving electrodes is developed. On each side, force are calculated and converted into moment, assuming point load radius $R_{c l}$. In equation (31), equation (30) is rearranged, considering the small displacement, $z_{0}>>$.

$$
\Delta M_{1} \approx-R_{c 1} \varepsilon\left(4 V_{B} U_{c}\right)\left[\frac{2}{3} \cdot \frac{\left(R_{f b o}^{3}-R_{f b i}^{3}\right) \cdot(\phi-\theta)}{\left(z_{0}\right)^{3}}\right]
$$

When displacement is controlled, displacement $z$ has very small values. Considering that $z_{0}$ is $3 \mu \mathrm{m}$ as a nominal point, $\mathrm{z}$ at equilibrium vibrates at almost $1 / 110$ of $z_{0}$, having a max value of $2.65 \times 10^{-2} \mu \mathrm{m}$. In a similar process, the moment generated by the compensator electrodes is developed as

$$
\Delta M_{1}^{\prime}=R_{c 1}^{\prime} \varepsilon\left(U_{c}\right)^{2}\left[\frac{2}{3} \cdot \frac{\left(R_{f b o^{\prime}}^{3}-R_{f b b^{\prime}}^{3}\right) \cdot(\phi-\theta)}{\left(z_{0}\right)^{3}}\right]
$$

As is known from equations (31) and (32), moments generated by each electrode can be corrected using a proper scaling because they have mutually opposite signs.

Using the equations developed, the error factor of the drift rate can be reduced by using the moment of the new sub-electrodes. Fig. 9 shows that compensating moment is applied to mitigate the result of displacement in the firstthird quadrant plane. Considering the characteristics of

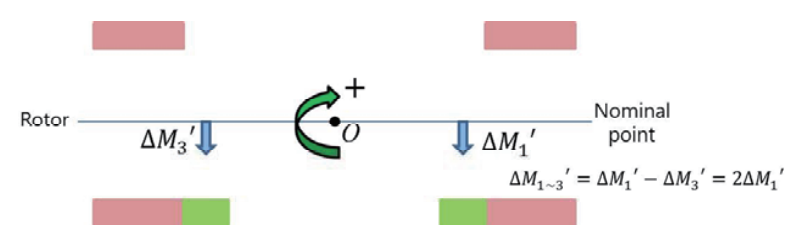

Fig. 9. Applying the point load moment dynamics.

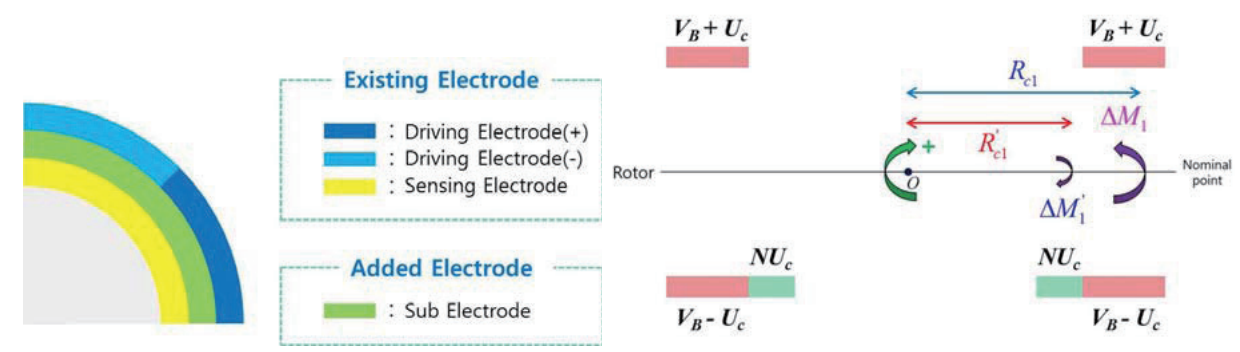

Fig. 8. One quadrant sub-electrode layout and cross sectional view of quadrants 1-3. 
the moment, the point load has a minus sign in the first quadrant and a plus sign in the third quadrant. However, the dynamic model in the third quadrant has $\phi$ and $\theta$ of opposite signs, and consequently the differential point load moment is generated as twice the single moment, $\Delta m_{1}^{\prime}$. Note that the same compensation moment is derived for the other quadrant pairs, i.e., the second-fourth quadrant pair in the vertical drift mitigation structure.

Moment generated by sub-electrodes $\left(\Delta m_{1}^{\prime}\right)$ is relatively smaller than moment generated by driving electrodes $\left(\Delta m_{1}\right)$. As observed from (31) and (32), moment generated by sub-electrodes is smaller than that from driving electrodes because coefficient of moment of driving electrodes have $4 V_{B}$ and the inner and outer radius from the center point are larger than those of compensating electrodes. Because of this, compensator electrode should have large area or high voltage should be applied. If area of compensator electrode is allocated too small, applied voltage may be not be acceptable, thus a proper design of area is essential. Considering various constraints on manufacturing and scaling factor for control purpose, compensator electrode are designed as in table 2 .

\section{Simulation of displacement control and analysis results}

A simulation study was performed to validate the performance of the designed drift rate compensator in a levitation gyroscope. As noted, the moment generated by the compensator electrodes is specifically smaller than that of driving electrodes due to short moment radius and smaller electrode area. A simple scaling compensation is suggested for drift mitigation, where a background rebalance control using a PID controller stabilizes the nominal suspended status.

Figure 10 shows the displacement control results by changing the compensator's gain with $0,8.5$, and 8.9.

Table 2. Size parameter of sub-electrodes

\begin{tabular}{|l|l|l|l|l|}
\hline & \multicolumn{2}{|l|}{ Previous model } & \multicolumn{2}{l|}{ Proposed model } \\
\hline \multirow{2}{*}{$F_{z}$ (Driving } & $R_{f b o}$ & $2990 \mu \mathrm{m}$ & $R_{f b o}$ & $2990 \mu \mathrm{m}$ \\
\cline { 2 - 5 } & $R_{f b i}$ & $1510 \mu \mathrm{m}$ & $R_{f b i}$ & $1510 \mu \mathrm{m}$ \\
\hline \multirow{2}{*}{$F_{z}^{\prime}$ (Sub Electrode) } & & & $R_{f b o}^{\prime}$ & $1500 \mu \mathrm{m}$ \\
\cline { 3 - 5 } & & & $R_{f b i}^{\prime}$ & $1000 \mu \mathrm{m}$ \\
\hline
\end{tabular}

The box with the dotted line is the error factor before compensation and box with the solid line is the error factor after compensation. As can be seen from the figure, as the scaling constant becomes larger, the amplitude of the error factor becomes smaller, until a compensator's stability margin is reached. If the compensation gain is above 9, moment generated by the compensator electrodes does not guarantee initial convergence during the transient period (i.e., after the compensator is on at $1.22 \mathrm{~s}$ ) and thus cannot satisfy drift mitigation. The excessive scaling gain becomes a trade-off between a fast convergence speed of the drift rate and steady state oscillation error. In Fig. 10, it can be seen that the steady state error is small enough, with a rising time less than $0.1 \mathrm{~s}$, which is satisfactory, considering MEMS levitation gyroscope performance.

Figure 11 shows the tilt angle transient about the $Y$-axis with a scaling gain of 8.9. The result corresponds with goal of

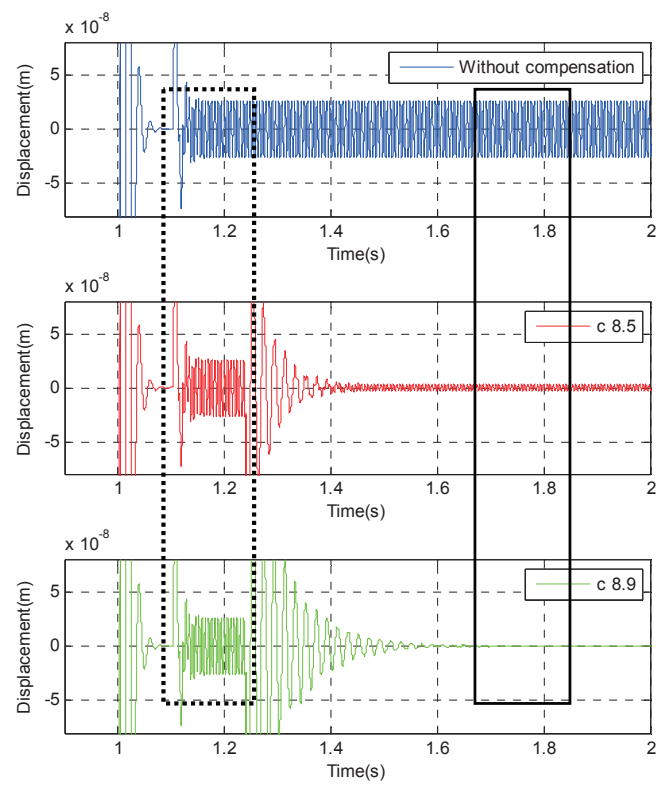

Fig. 10. Displacement result of simulation (compensation gain $0,8.5$, 8.9).

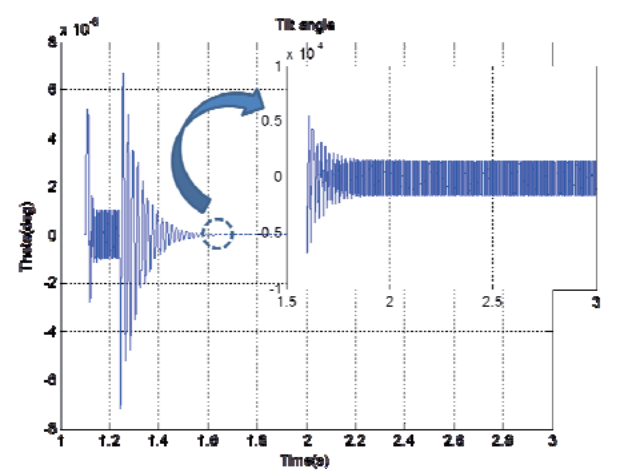

Fig. 11. Y-axis tilt angle - result of simulation (compensation gain 8.9). 
this paper, to stabilize tilt angle drift. In the stable section, the amplitude from drift is $4.11 \times 10^{-4} \mu m$. This shows that the subelectrodes stabilize the displacement at $\sim 1 / 64$, compared with the existing amplitude of oscillation, $2.65 \times 10^{-2} \mu \mathrm{m}$. Similarly, in Fig. 11, the maximum tilt angle converges to $1.57 \times 10^{-50}$, which provides the proposed design to stabilize the tilt angle at $\sim 1 / 66$, compared with non-compensation result of $10^{-30}$. It can also be seen that the bias and control voltage for the proposed algorithm satisfy the typical requirement of analog electronics, showing its practical feasibility. Consequently, the proposed compensator design has the possibility of reducing the amplitude of drift error factors caused by inevitable mass unbalance in the levitated gyro. Also, by refining the size of area and applied voltage, further improvement in the amplitude mitigation with sufficient transience will be achieved.

\section{Conclusions}

In this paper, to realize levitated gyro performance under practical error factors, a vertical dynamic model was established and investigated, such that displacement converged within a suitable control voltage at the nominal point using a controller based on a linearized model. Also, a drift rate formula was developed under an assumption and initial values considering the mass-unbalance of the rotor. The drift effect with the displacement control was analyzed quantitatively for performance validation.

The unexpected motion of the wobbling effect occurs through high rotational velocity and can have a critical effect on the system. In case of a levitated gyro, a factor that threatens the stability of displacement should be analyzed and stabilized using correction or control, because control of displacement is the key to the accurate operation of the levitated gyro. In this paper, to solve the problem, we introduced the concept of sub-electrodes for compensation purposes. Having shown the usefulness of the designed model, a new method to increase the stability of displacement is proposed.

In future work, the drift rate of the rotor will be verified by performing experiments with a real rotor and results from theoretical calculations will be compared with real data quantitatively. Using these results, the suitability of the assumptions will be evaluated. Also, it will be verified that stability of displacement is increased by applying the subelectrodes.

\section{Acknowledgements}

This research was supported by a grant to the MEMS Research Center for National Defense funded by the Defense Acquisition Program Administration and partially supported through a research grant provided by the Korean Ministry of Science, ICT \& Future Planning (MSIP) in 2013 (2013R1A1A100640).

\section{References}

[1] Q. J. Xiao, W. Y. Chen, G. Ma, F. Cui, S. Y. Li, and W. P. Zhang, "Simulation of Levitation Control for a Micromachined Electrostatically Levitated Gyroscope," IEEE-NEMS 2009, January 2009, pp. 160-163.

[2] L. Yan-zhu, and X. Yun, "Drift Motion of FreeRotor Gyroscope with Radial Mass-Unbalance," Applied Mathematics and Mechanics, vol. 25, July 2004, pp. 786-791.

[3] R. Houlihan, and M. Kraft, "Modelling of an accelerometer based on a levitated proof mass," Journal of Micromechanics and Microengineering, vol. 12, May 2002, pp. 1-8.

[4] F. Han, Q. Wu, and R. Zhang, "Modeling and Analysis of a Micromotor with an Electrostatically Levitated Rotor," Chinese Journal of Mechanical Engineering, vol. 22, No. 1, February 2009.

[5] T. Murakoshi, Y. Endo, K. Fukatsu, S. Nakamura, and M. Esashi, "Electrostatically Levitated Ring-Shaped RotationalGyro/Accelerometer," Japanese Journal of Applied Physics, vol. 42, April 2003, pp. 2468-2472.

[6] B. Damrongsak, M. Kraft, S. Rajgopal, and M. Mehregany, "Design and fabrication of a micromachined electrostatically suspended gyroscope," Proceddings of the Institution of Mechanical Engineers Part C-Journal of Mechanical Engineering Science, vol. 222, June 2007, pp. 53-63.

[7] M. V. Gindila, and M. Kraft, "Electronic interface design for an electrically floating micro-disc," Journal of Micromechanics and Microengineering, vol. 13, June 2003, pp. 11-16.

[8] F. L. WALKER, "An Automatic Gyro Mass-Unbalance Measuring Technique," IEEE Transactions on Military Electronics, vol. 7, Jan 1963, pp. 36-40.

[9] B. Damrongsak, "Development of a Micromachined Electrostatically Suspended Gyroscope", Doctoral Thesis, School of Electronics and Computer Science, University of Southampton, Apr 2009. 